\section{SEEING EVER-SMALLER WORLDS*}

\section{By SIR LAWRENCE BRAGG, O.B.E., F.R.S.}

$\mathrm{T}$ HE limit to microscopic resolution is set by the wave-length of light. The degree of indistinctness of an object when seen under high magnification can be estimated if we picture the light which is traversing the instrument reversed in its direction. Instead of light waves scattered by the object passing on to build up the image, suppose corresponding light waves to start from the image and proceed towards the object. The pattern which they build up in the object plane will be, on a very reduced scale, what is actually seen when looking through the microscope, and since the local ether storms which they produce must be at least about a wave-length in each dimension, all sharp edges or fine detail in the object must be blurred to this extent. It is as if we were trying to paint a picture with a brush which gives a broad line, the breadth being the wave-length of light. Detail on a finer scale than a brush mark must escape its coarse stroke. Using the shortest ultra-violet waves for which transparent lenses are available, and transparent media of high refractive index in which to immerse the object, the limit of resolution cannot be pushed beyond $1000 \mathrm{~A}$. $\left(10^{-5} \mathrm{~cm}\right.$. or $\left.1 / 10 \mu\right)$. As Abbe pointed out in his treatment of the microscope, this is an absolute barrier set by Nature, and if it is to be surpassed it must be by employing some quite new principle.

All bodies cease to be transparent for ultra-violet light more than an octave beyond the end of the visible spectrum. It is not until we reach the $\mathrm{X}$-ray region that waves are again able to traverse matter to an appreciable extent. This opens up the possibility, which has been exploited in X-ray analysis, of getting information about the form of a body by observing the way it scatters X-rays. Convenient $\mathrm{X}$-ray wave-lengths range from $2 \cdot 0$ to $0.5 \mathrm{~A}$., and so lie in a region 4,000 times smaller than that of light waves. Resolution is correspondingly increased, and is in fact sufficient not only to separate atoms in a solid but even to give considerable information about the distribution of scattering matter in an atom. We have, however, to pay a heavy price for this gain in resolving power. We cannot form an image of an object illuminated by X-rays. Even if the technical difficulties of designing some type of reflecting lens capable of focusing X-rays could be overcome, the amount of scattered X-ray energy is inadequate. Consider, for example, a minute object, about $1000 \mathrm{~A}$. each way, illuminated by ordinary light. Under intense illumination the amount of light scattered by such a body is sufficient for photographic recording but is nearing the limit. Yet such a body contains 30 million atoms, and when it is remembered that the most intense $\mathrm{X}$-ray beam has far less energy than we are able to get in a light beam, it will be realized that even if the $\mathrm{X}$-rays scattered by single atoms could be focused, the energy would be far too small to be observable.

It is therefore not possible to bring the X-rays from each point of the object to corresponding points of an image and so make direct use of their resolving power. It is only possible to observe the intensity of $\mathrm{X}$-radiation scattered in any given direction by the object, and to get all the information we can about

* Friday evening discourse delivered at the Royal Institution on March 12. the object by interpreting such observations. This limitation decides the peculiar nature of X-ray analysis. In the first place, an object of irregular form gives a most complicated distribution of scattered rays. It is only when the object is a regular repetition of a unit of pattern that the scattered rays are diverted to certain directions, and that it is possible to associate in a simple way the strength of the scattered rays with the form of the unit. Hence X-ray analysis finds its most ready application to the examination of crystal structures where such regular patterns exist. Further, since we can only measure the intensities of the scattered beams, vital information about the form of the object is lost. When an object is viewed by the microscope, light is scattered in different directions by its various parts. These light waves pass through the instrument, and interfere in the image plane to build up a light distribution which corresponds, within its limit of resolution, with the object. The form of the image is determined, not only by the amplitudes of the waves scattered in different directions, but also by their relative phases, since the interference depends on these phases. In $\mathrm{X}$-ray analysis, we can measure the amplitudes of the waves scattered in different directions, but their relative phases are not observable. Hence we have not got enough information to reconstruct the object. An infinity of objects, deduced by assigning any arbitrary phases to the scattered waves, would account for any one set of $\mathrm{X}$-ray results.

The interpretation of X-ray diffraction is therefore like that of solving a cross-word puzzle. We cannot proceed directly; we must guess, and test whether we have guessed correctly. We know that the actual object, a crystal structure, consists of a regular array of certain groups of atoms. We know how much each atom scatters X-rays, and experience with other analyses teaches us much about the probable configuration of the atomic groups, what distance apart they are likely to be, and which are likely to be neighbours. Armed with such experience, possible configurations are tested to see whether they explain all the X-ray results, until one is found which satisfies every check. X-ray analysis has proceeded, as experience has accumulated, from simple structures where the alternatives are few to highly complex ones.

There is a simple optical experiment which illustrates the difficulty of X-ray analysis, and makes a striking demonstration. A cross-grating pattern, with about fifty repetitions of the unit to a centimetre each way, is illuminated by parallel monochromatic light and viewed by a microscope. A convenient object is a series of circular transparent holes, made as a print from the type of process plate used in half-tone reproduction. When the microscope is accurately focused, these holes are seen in their true form. Now the light message received by the microscope under these conditions is a series of parallel beams, the cross-grating spectra. The intensities and directions of these beams remain the same whatever the distance of the objective from the cross-grating. These beams therefore continue to build up an image when the objective is moved away from or towards the object, out of the focused position. The only variation when the objective is moved is in the relative phases of the parallel beams, which on passing through the instrument build up the image. The racking of the microscope out of focus affects the path-lengths of the beams to a different extent. We therefore see a different image for every position of 
the objective. The image may be regarded as built up of a two-dimensional Fourier series of fringes, and these fringes become very obvious when so thrown out of phase that they no longer build up the true image. If the source of illumination is a mercury vapour lamp, the various wave-lengths build up image patterns of considerable beauty, which resemble a brilliant series of tartan patterns. Every image is realistic, and we cannot focus the microscope unless we know what we ought to be seeing - in this case, a series of round holes. In a precisely similar way, the phases are uncertain in X-ray analysis, and we can only 'focus' because we know we ought to be seeing atoms.

In contrasting $\mathrm{X}$-rays and light, we see, therefore that $X$-ray analysis attains very great resolving power, but is difficult to apply except to structures which are regular repetitions of a simple unit. In the case of light, the complexity of the 'geography' of the specimen presents no difficulty because we can form images, but there is a serious limitation to the resolving power. To what extent has the gap been filled?

X-ray analysis has been extended to ever more complex structures. A classic example is the work of J. M. Robertson on organic molecules. If by getting a first rough approximation to a structure we can determine the phases of the scattered X-ray beams, we can then use the measured intensities to determine the amplitudes of these beams, and so get complete information about the form of the object. The problem is considerably simplified by the fact that in most crystals the unit of pattern has a centre of symmetry when projected on certain planes. The phase is then positive or negative. To put this more precisely, the wave scattered by the unit of pattern as a whole must by symmetry be either in the same phase as, or opposite phase to, that scattered by a point at its centre. By amplitude is meant the amplitude of the resultant wave as compared with that scattered by a pattern of single electrons at the centres. By summing a double Fourier series with these phases and amplitudes, a map of the electron density in the crystal is obtained which is a true 'X-ray image' of the structure projected on a chosen plane.

$\mathrm{X}$-ray analysis is being extended, however, to even more complex fields. Crystals of certain proteins give $\mathrm{X}$-ray diffraction pictures which show that they are extremely regular. Bernal first obtained such pictures, and the work has been carried on by Fankuchen, Mrs. Hodgkin, Perutz and others. The X-ray pictures show a wide array of very fine spots ; and if only one could deduce the phases, the intensities of the spots could be used, as in Robertson's work on organic molecules, to build up an X-ray image of a protein. The difficulty of guessing these phases is formidable. A molecule of hæmoglobin, studied by Perutz, has a molecular weight of about 70,000 . There are encouraging signs, however, that progress is not impossible. Perutz has discovered a method of making the crystal structure shrink without distortion of the hæmoglobin molecules, which in the fully expanded crystal are separated by sheets of the liquid bathing the crystal. Now if we had a complete plot of the scattering power of a centro-symmetrical molecule for different directions of scattering, we could deduce the phases. They are either positive or negative, and as the direction varies the scattering power must go through a zero value in passing from one to the other. Hence the positive and negative regions of scattering are separated by nodes as in a vibration problem, and with the whole picture before us we could take the central region to be positive, and proceeding outwards change the sign every time we pass through a node. Ordinarily this is not possible, because the molecular scattering factor is sampled only at certain points, namely, the spectra determined by the grating constants of the crystal. When the grating constant itself can be varied, however, as in the case of hæmoglobin, the spectra sweep through the molecular scattering distribution and fall to zero in traversing the nodes. In this way Perutz has been able to trace a number of the nodes, and has reduced the problem of deciding between positive and negative for the inner spots to a few alternatives. Only one of these alternatives turns out to give a reasonable answer. Hence Perutz has been able to get an X-ray image of the protein molecule. This image is still very imperfect, corresponding to a resolving power of about $7 \mathrm{~A}$., but the molecule is beginning to take shape and the process can be extended.

Another example of larger scale structures attacked by X-ray analysis is afforded by the studies of more or less regular fluctuations of the perfect crystal array such as occur in certain alloy structures. Preston's work on age-hardening, Bradley's work on the permanent magnet alloys, and the study by Lipson and others of 'sidebands' are cases in point. When an optical grating is ruled by a faulty engine, so that periodic disturbances of its regularity are produced, the result is a series of false lines or 'ghosts' in the neighbourhood of each spectral line. Similarly, when a crystal lattice is distorted in a harmonic way, each X-ray diffraction beam has ghost beams in its neighbourhood. The separation between the main line and the ghost is a measure of the period of fluctuation. Such fluctuations occur in alloys in a metastable state, when full separation into phases has not been attained. Periods of the order of $100 \mathrm{~A}$. have been studied in this way.

The electron microscope, which has been developed in recent years, is of such absorbing interest because it fills in the wide gap between analysis by light and by X-rays. Experiments in the development of the instrument were begun by Knoll and Ruskin in Germany in 1932. An account of its principles will be found in an address given to the Royal Institution in May 1940 by Prof. L. C. Martin (see Nature, August 31, 1940, p. 288). The electron microscope forms images like the ordinary microscope, while its resolving power is about forty times as great. It owes this power to two factors. In the first place, the Abbe limit, or limit to resolving power due to wave-length, is extremely small. The wave-length of 60 kilovolt electrons is $0.5 \times 10^{-9} \mathrm{~cm}$. as compared with $0.5 \times 10^{-4} \mathrm{~cm}$. for yellow light. A corresponding increase in resolving power is not attained, because the numerical aperture of the magnetic or electrostatic lenses employed by the electron microscope has to be made extremely small to avoid aberrations. However, even with the small apertures ordinarily used, the Abbe limit of the instrument is about $10^{-7} \mathrm{~cm}$. or $10 \mathrm{~A}$. Certain imperfections limit the resolving power of the best instruments yet constructed to 30-40 A., which approaches the Abbe value. Causes of imperfection are the aberrations and not completely symmetrical fields of the lenses and the fluctuations in voltage at the electron source. A resolving power of $100 \mathrm{~A}$. is attained without serious difficulty. This high resolving power would by itself not be of service, were it not for another fortunate 
factor. The intensity of illumination by the electron beam is immensely greater than that for the brightest possible light source. Gabor estimates it as being in common practice $10^{6}$ times as great as the intensity of the sun's image. Hence extremely small masses of matter containing a few thousand atoms scatter sufficient energy to produce an impression on a photographic plate.

The principle of the instrument is very simple. A co-axial magnetic or electric field acts as a lens for a beam of electrons of given velocity. By suitable design the focal length of such lenses can be reduced to a few millimetres. A first condenser lens concentrates 50,000-volt electrons on the object. After traversing it, they pass through an objective lens which forms an image magnified 100 or 200 times. This is viewed in a fluorescent screen with a fine hole at its centre, and a chosen portion of the image is brought over the hole. This portion is again magnified to a similar extent by a final lens, and viewed on a screen or photographed.

The objects must be extremely thin, less than $1,000 \mathrm{~A}$. in thickness, and are of course in a vacuum. They may be deposited on a very fine pellicule of collodion which is laid on a plate pierced with fine holes. Focusing is achieved by adjusting the current in the magnetic lens. It is impossible to view the surface of a solid directly, since transmission must be used. It is possible, however, to examine the contours of a surface, such as that of an etched metal, by spreading a fine film of collodion over the surface and stripping it off, when it retains an impression of the hollows and ridges.

The War has retarded the applications of this instrument which would undoubtedly have otherwise been made, and we can confidently expect that it will open up fascinating new fields in future. On one hand, it gives greater definition to objects that can be seen under the microscope, and on the other it attains a resolving power in the $100 \mathrm{~A}$. region, which is of the same order as that of the larger. scale structures studied by X-rays, so that we now have instruments capable of use in the whole range between the visible and atomic dimensions.

\section{PRESENTATION OF SCIENCE TO A GENERAL PUBLIC*}

\section{By DR. ARNOLD RAESTAD \\ Formarly Minister of Foreign Affairs to the Norwegian Government}

A LTHOUGH I am not a scientific man myself, A it has fallen to my lot to be connected with certain aspects of the problem of the exposition of science. I have been engaged in examining the problem of how to organize better the presentation of the methods and results of science throughout the world or, in other words, the international dissemination of science. After three or four years of preparatory work, in 1938, the League of Nations, through the International Institute of Intellectual Co-operation in Paris, appointed a committee of scientific men, presided over by myself, to report on the matter. Before I say more about the committee and

* Substance of a paper read on March 20 before the Division for the Social and International Relations of Science of the British Association at the Conference on "Science and the Citizen". its work, it may be instructive to record, very briefly, the preliminary inquiries which led to its appointment.

The Assembly of the League of Nations had interested itself, in a couple of resolutions, in the use of broadcasting to promote the cause of peace and to familiarize nations with the culture of other nations. In 1936, an international convention for the use of broadcasting in the interest of peace was concluded. Later, the Institute of Intellectual Co-operation asked me to consider what subjects it might most appropriately pick out for an organized effort in this connexion. The Institute referred more particularly to the cause of peace and the work of the League, and to literature and culture generally, as promising objects for an internationally organized effort. I had to report that, in my opinion, neither the peace work of the League of Nations nor the knowledge of the literature of other peoples lent themselves very well to such a co-ordinated international effort.

But there was another field in which the practical broadcaster knew there was something to be donescience. For some reason or other, the attention of the statesmen of the League had not been focused on the need to disseminate more perfectly a knowledge of science-to publicize science. In most countries of Western Europe, despite the vastly increased effectiveness of the common media of information, the information at the disposal of the general public is less adequate, both as regards the progress of science and as regards current topics involving the existence of a scientific problem, than was the case thirty or forty years ago. The Press, filling its public to the saturation point with news and political views, has only a languid interest in the results and methods of science. The documentary film and the radio have not, in actual practice, filled the gap. As for the radio, it has no doubt rendered great services here, for listeners are easily attracted by great scientific names or fascinated by discoveries in biology or astronomy. But generally speaking, according to reports from many countries, the radio has not given what might have been expected, for a number of reasons. Outstanding men of science very rarely combine the qualities which make a good and attractive speaker on the radio; further, no single country has at its disposal first-rate men in all branches of science.

The Institute of Intellectual Co-operation, in view of the preliminary reports thus gathered, appointed in 1938 a committee of scientific men to consider means of improving, by organizational measures, the way in which the results and methods of science are now presented to a general public. The members of the committee were Dr. Julian Huxley, M. Henri Laugier, Dr. Clarence C. Little, my compatriot the late Prof. Sem Saeland, a Swiss, a Dutch and a Belgian man of science. All modern media of current presentation were considered in turn-lectures, Press, films, exhibitions, radio. The problem was approached from the point of view of science as well as from that of society, and from the point of view of the individual scientific worker as well as from that of organized and collective science. The committee was unanimous in stating as its opinion that the situation called for reforms of an organizational character, that these reforms would have to be carried out, if at all, by existing scientific bodies, and that the organization involved would have to be international in its scope and conceptions. 